\title{
Author Correction: Solar methanol energy storage
}

Athanasios A. Tountas, Geoffrey A. Ozin (1) and Mohini M. Sain

Correction to: Nature Catalysis https://doi.org/10.1038/s41929-021-00696-w, published online 18 November 2021.

In the version of this article, there was an error in Fig. $2 \mathrm{~b}$. The $9,900 \mathrm{~h}^{-1}$ space velocity data were plotted against the wrong $x$-axis values (originally 100, 70, 50,30, 10\% rather than the corrected 70, 50, 30, 20, 10\%). The plot has been replaced. Further, in the explanation below equation (3), the text describing variable " $a$ " has been expanded to include catalytic activity for the CZA catalyst, and now reads “.... and the variable $a$ denotes the exposed metallic surface area or the catalytic activity ${ }^{70 "}$

The changes have been made to the HTML and PDF versions of the article.

Published online: 24 December 2021

https://doi.org/10.1038/s41929-021-00731-w

๑) Springer Nature Limited 2021 